\title{
Malakoplakia of the Prostate as a Mimicker of Prostate Cancer on Prostate Health Index and Magnetic Resonance Imaging-Fusion Prostate Biopsy: A Case Report
}

\author{
Nathaniel H. Heah, MBBS, MRCSEd, Teck Wei Tan, MBBS, MRCSEd, MMed (Surgery), FAMS (Urology), \\ and Yung Khan Tan, MBBS, MRCSEd, MMed (Surgery), FAMS (Urology)
}

\begin{abstract}
Background: Isolated malakoplakia of the prostate is a rare inflammatory condition that has been clinically mistaken for prostatic malignancies. The development of Prostate Imaging Reporting and Data System (PIRADS) classifications, and Prostate Health Index (PHI) has led to more accurate diagnosis of clinically significant disease and stratification of patients that may be at risk of prostate cancer.

Case Presentation: We present a case of a 75-year-old male who was on follow-up with our hospital for elevated prostate specific antigen (PSA). He was admitted for an episode of urosepsis, which was treated with antibiotics and subsequently underwent further workup and was found to have a raised PHI, as well as a high PIRADS classification and was later found to have malakoplakia based on histology of prostate tissue obtained during targeted magnetic resonance imaging (MRI)-guided fusion prostate biopsy.

Conclusion: To our understanding, this is the first case where a prostate lesion has been labeled as a PI-RADS 5 lesion, with elevated PHI that has subsequently been proven histologically to be malakoplakia. An important possible confounder is the interval between the MRI and the episode of urosepsis and it is well known that urosepsis can affect the PSA and MRI result. We present this case to highlight the potential for a false diagnosis of prostate cancer, in spite of laboratory and radiological findings.
\end{abstract}

Keywords: malakoplakia, Prostate Health Index, MRI-fusion biopsy, PI-RADS

\section{Introduction and Background}

M ALAKOPLAKIA IS A rare inflammatory condition thought to develop from chronic Escherichia coli infection. While malakoplakia is seen throughout the genitourinary tract, isolated malakoplakia of the prostate is rare, ${ }^{1}$ and has been clinically mistaken for prostatic malignancies. Definitive diagnosis is usually from histological confirmation by prostatic biopsy. ${ }^{1}$ Malakoplakia has even been mistaken for locally advanced prostate cancer on multiparametric magnetic resonance imaging of the prostate (mpMRI Prostate). ${ }^{1}$

Diagnosis of prostate cancer has developed over the last few years and the advent of the prostate imaging reporting and data system (PI-RADS) classifications, ${ }^{2}$ as well as the use of mpMRI prostate for MRI-guided fusion prostate biopsies have led to more accurate diagnosis of clinically significant disease. Additionally, the use of Prostate Health Index (PHI) has helped to better stratify patients that may be at risk of prostate cancer. ${ }^{3}$ We present a case of elevated prostate specific antigen (PSA) in a 75-year-old man who was seen for further urological work-up, was found to have a raised PHI, as well as high PI-RADS classification, and was later diagnosed to have malakoplakia based on the histology of prostate tissue obtained during targeted MRI-guided fusion prostate biopsy.

\section{Presentation of Case}

Clinical history

A 75-year-old male was on follow-up with our hospital for elevated PSA levels of $16.6 \mu \mathrm{g} / \mathrm{L}$ on screening. Examination

Department of Urology, Tan Tock Seng Hospital, Singapore, Singapore.

(c) Nathaniel H. Heah et al. 2017; Published by Mary Ann Liebert, Inc. This is an Open Access article distributed under the terms of the Creative Commons Attribution License, which permits unrestricted use, distribution, and reproduction in any medium, provided the original work is properly cited. 
showed an enlarged prostate with no nodules. He was asymptomatic and was offered a standard 12-core systematic needle biopsy of the prostate in March 2016. Histology at the time showed benign prostatic tissue in all cores. He was admitted in November 2016 for sepsis secondary to $E$. coli urinary tract infection (UTI) complicated by $E$. coli bacteremia, for which he was treated with intravenous antibiotics and subsequently recovered.

\section{Diagnosis}

His PSA levels were monitored and continued to rise, prompting a second biopsy. He was offered a prebiopsy mpMRI prostate for possible MRI-guided fusion prostate biopsy in December 2016 as well as PHI testing. mpMRI was obtained in January 2017 using a 3T MRI scanner without an endorectal coil. This MRI demonstrated a well-circumscribed right apical homogeneous, hypointense, peripheral zone lesion on T2-weighted imaging measuring $2.8 \mathrm{~cm}$. The lesion was also markedly hyperintense on high $b$-value diffusionweighted imaging, and hypointense on the apparent diffusion coefficient map. These findings were suggestive of a PI-RADS 5 lesion. A second $1.2 \mathrm{~cm}$ lesion in the left mid-gland peripheral zone was also classified as a PI-RADS 4 lesion. His repeat PSA was $21.76 \mu \mathrm{g} / \mathrm{L}$, and PHI was 187 (Fig. 1a-d).

MRI-ultrasound fusion biopsy was performed using the UroNav $^{\circledR}$ system. The prostate volume was measured using transrectal ultrasound as $46 \mathrm{~cm}^{3}$. In brief, the MRI images with targets were marked by a dedicated uroradiologist and loaded onto the system beforehand. The MRI images were then overlaid onto real-time ultrasound images from the transrectal probe, allowing for three-dimensional reproduction of the prostate with the ultrasound and subsequent targeting and tracking of biopsy tracts (Fig. 2).

A total of three cores from each target were obtained, and a systematic 12-core biopsy was also performed at the same setting. Ten out of 12 cores from the systematic biopsy, as well as all six targeted cores, showed intracytoplasmic blue-gray bodies called Michaelis-Gutmann bodies (MGBs), some showing "owl's eye" appearance. The tissue also featured infiltrates of macrophages with lymphocytes, plasma cells, and occasional multinucleated giant cells. Immunostains for cytokeratin AE $1 / 3$ showed no evidence of invasive carcinoma. Based on these findings, the diagnosis of malakoplakia of the prostate was made (Fig. 3a, b).

\section{Discussion and Literature Review}

Malakoplakia has been known to mimic malignancy in the prostate. It is in fact a chronic inflammatory condition that usually involves the genitourinary system. Histologically it is characterized by the presence of von Hansemann macrophages including MGBs. It is seen most commonly in patients with UTIs that subsequently grow $E$. coli $\mathrm{sp}$. in urine cultures.

This case continues to highlight the rare differential diagnosis of malakoplakia when assessing a patient for prostate malignancy. To our understanding, this is the first case, where a prostate lesion has been labeled as a PI-RADS 5 lesion, with elevated PHI that has subsequently been proven histologically to be malakoplakia.

The use of mpMRI prostate is now accepted in the diagnosis and staging of prostate cancer. More recently, with the development of targeted biopsies and PI-RADS v2, ${ }^{2}$ which has been shown to have a sensitivity of $95 \%{ }^{4}$ for prostate cancer foci of $\geq 0.5 \mathrm{~mL}$, the aim of this scoring system is still to avoid unnecessary biopsies.

In addition, PHI has shown to have some usefulness in avoiding unnecessary biopsies in patients with normal digital rectal examination and PSA from 10 to 20. PHI is a mathematical formula that combines total PSA, free PSA, and [-2] form of proPSA (p2PSA) to predict the overall risk and risk of high-grade prostate cancer on biopsy. It has also been shown to predict the likelihood of progression during active surveillance.

This case is a unique case, where malakoplakia presents as a mimicker of prostate malignancy on both laboratory and radiological investigations and adjuncts that have been validated in the diagnosis of prostate cancer.
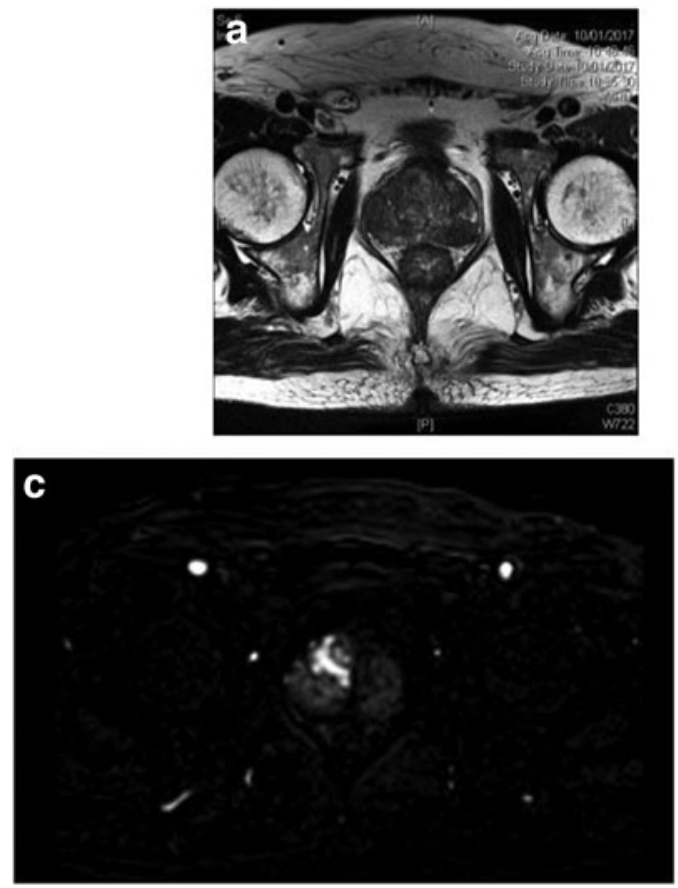
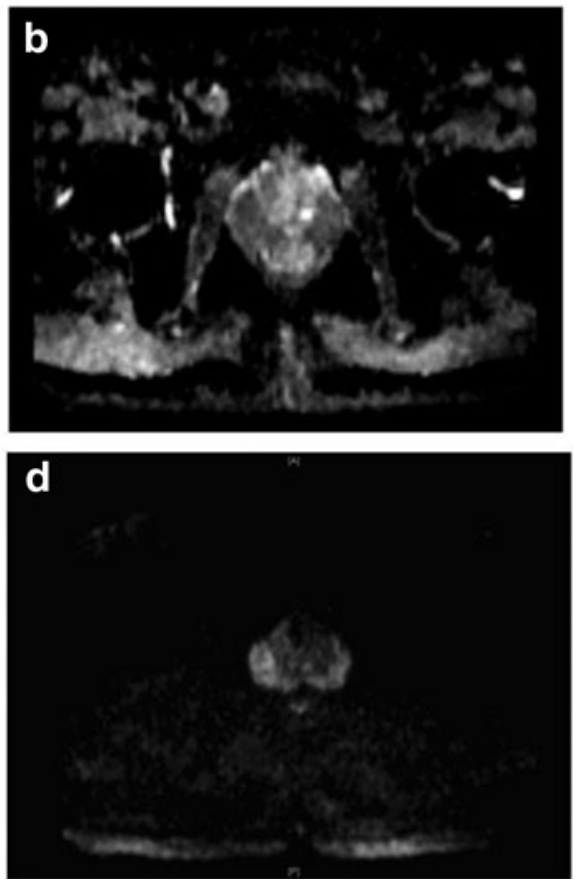

FIG. 1. (a) Hypointense bilateral peripheral zone lesion on T2-weighted image $(2.8 \mathrm{~cm}$ right side, $1.2 \mathrm{~cm}$ on left side). (b) Hypointense lesions corresponding to T2weighted images on apparent diffusion coefficient map. (c) Early enhancement of right peripheral zone lesion on dynamic contrast enhancement imaging. (d) Markedly hyperintense bilateral peripheral zone lesions on high $b$-value diffusion-weighted imaging. 


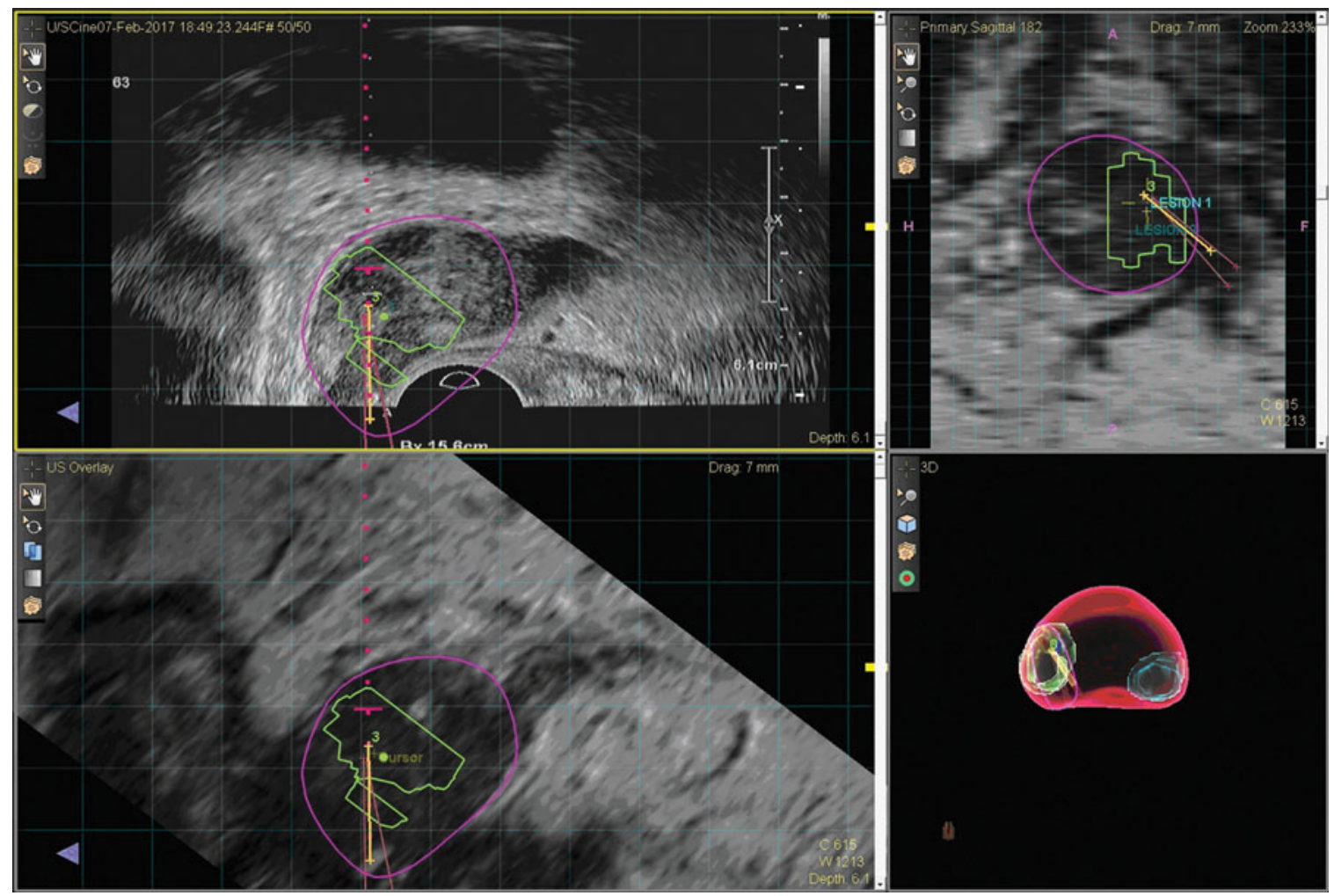

FIG. 2. Biopsy with UroNav system showing 3 cores through targeted lesion.
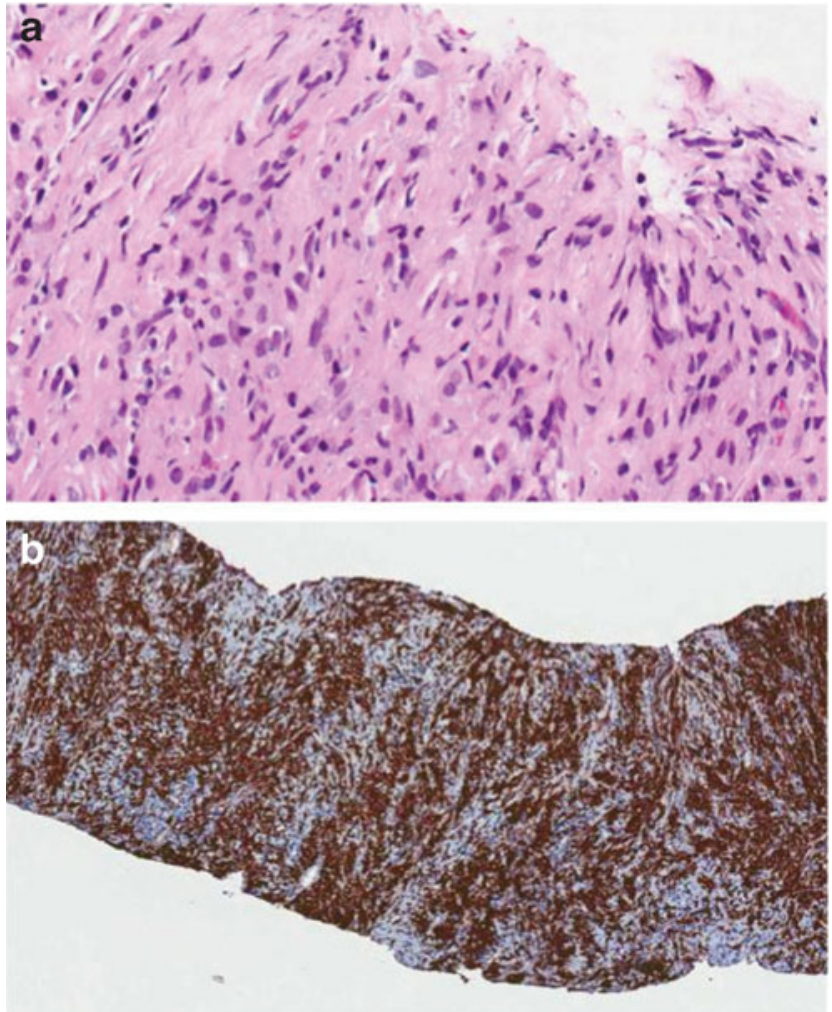

FIG. 3. (a) Michaelis-Gutmann bodies seen in the histology specimen. (b) CD164 stain showing histocytes throughout core specimens.
An important possible confounder is the interval between the MRI and the episode of urosepsis. There were at least 6 weeks between the patient's admission for urosepsis and his MRI; however, this may have been too short a period. The PHI was also done at the same time as the MRI and was found to be extremely high. This highlights the significance of having an appropriate interval before performing mpMRI and PHI after an episode of urosepsis. It is well known that urosepsis can affect the PSA and mpMRI result, and the timing of these investigations could have been a contributing factor to the impression of high-risk cancer; however, although there is an agreed time interval between prostate biopsy and mpMRI, which is usually 8 weeks, there is currently no literature on the appropriate time interval between an episode of urosepsis, and a subsequent mpMRI.

The patient's history of a recent $E$. coli UTI may have alluded to the possible diagnosis; however, this would not have changed his work-up in any way.

\section{Conclusion}

We present this case to highlight the potential for a false diagnosis of prostate cancer, in spite of laboratory and radiological findings.

\section{Author Disclosure Statement}

No competing financial interests exist.

\section{References}

1. Dale RT, Metcalfe M, Chang S, et al. Malakoplakia of the prostate masquerading as locally advanced prostate cancer on mpMRI. Can Urol Assoc J 2015;9:E910-E912. 
2. Vargas HA, Hötker AM, Goldman DA, et al. Updated prostate imaging reporting and data system (PIRADS v2) recommendations for the detection of clinically significant prostate cancer using multiparametric MRI: Critical evaluation using whole-mount pathology as standard of reference. Eur Radiol 2016;26:1606-1612.

3. Lazzeri M, Haese A, de la Taille A, et al. Serum isoform [-2]proPSA derivatives significantly improve prediction of prostate cancer at initial biopsy in a total PSA range of 2-10 ng/ ml: A multicentric European study. Eur Urol 2013;63:986-994.

4. Loeb S, Catalona WJ. The Prostate Health Index: A new test for the detection of prostate cancer. Ther Adv Urol 2014;6:74-77.

Address correspondence to: Nathaniel H. Heah, MBBS, MRCS Department of Urology Tan Tock Seng Hospital 11 Jalan Tan Tock Seng, 308433 Singapore

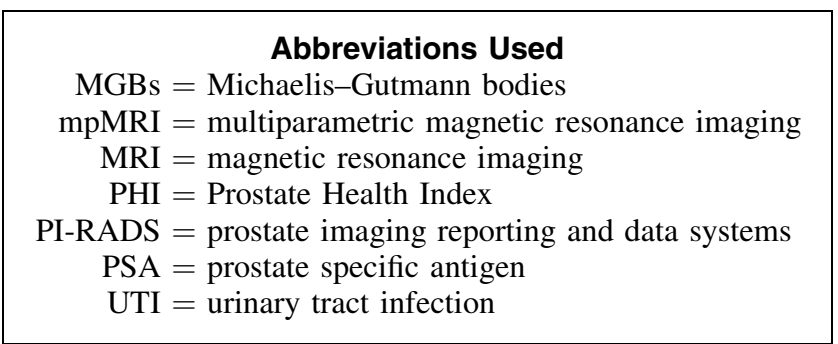

Cite this article as: Heah NH, Tan TW, Tan YK (2017) Malakoplakia of the prostate as a mimicker of prostate cancer on Prostate Health Index and magnetic resonance imaging-fusion prostate biopsy: a case report, Journal of Endourology Case Reports 3:1, 74-77, DOI: 10.1089/ cren.2017.0030.

E-mail: nathaniel.heah@mohh.com.sg 